\title{
Therapie von Tendinopathien mit Platelet-Rich Plasma?
}

\author{
Die Therapie mit Platelet-Rich Plasma (PRP) ist modern und \\ umstritten zugleich. Diese Meta-Analyse von randomisierten \\ und kontrollierten klinischen Studien stellt den aktuellen Stand \\ der Forschung dar. Aus dieser wissenschaftlichen Aufarbeitung \\ ergeben sich klare Handlungsanweisungen für die Praxis. \\ Fitzpatrick J, Bulsara M, Zheng MH. The Effectiveness of Platelet-Rich Plasma in the \\ Treatment of Tendinopathy: A Meta-analysis of Randomized Controlled Clinical Trials. \\ Am J Sports Med 2016; DOI: 10.1177/0363546516643716
}

Die Autoren haben eine Meta-Analyse aus randomisiert-kontrollierten Studien in der PRP-Therapie von Tendinopathien durchgeführt. Die Datenerhebung fand zwischen 03/2012 und 08/2015 statt.

\section{Methodik \\ $\nabla$}

18 Studien mit 1066 Patienten wurden nach PRISMA (Preferred Reporting Items for Systematic Reviews and Meta-Analyses) Guidelines mit Erfüllung des PRISMAIPD Statements eingeschlossen. Ausgewertet wurden randomisierte Studien, die mittels Injektion von PRP oder anderer autologer Blutprodukte Tendinopathien therapierten (siehe 0 Tabelle 1). Diese Blutprodukte konnten aus Vollblut, PRP oder „platelet-poor plasma“ (PPP) oder auch „autologous conditioned plasma“ (ACP) bestehen. Es wurde jede Dosierung und Anzahl sowie jedes Volumen an intrawie auch peritendinösen Injektionen zugelassen. Ausgeschlossen wurden Studien mit zusätzlicher Operation oder Weichteilverletzungen neben der Tendinopathie, wie zum Beispiel Muskel- oder Bandverletzung. Der primäre Zielparameter war die Änderung der Schmerzintensität. Eine Follow-Up-Zeit von mindestens 3 Monaten wurde vorausgesetzt. Zusätzlich wurde das Follow-Up nach 6 und 12 Monaten berücksichtigt. Pro Studie wurde jeweils ein Schmerz-Score eingeschlossen. Mögliche Bias wie Randomisierungsprozess, Ausschluss durch Patientenverzug, Verblindung der Patienten/Untersucher und finanzielle Unterstützung durch Firmen wurden beschrieben. Die Autoren haben die Gefahr für Bias in 3 Abstufungen zwischen niedriger Gefahr für beeinflussende Bias, unsicher und hoher Gefahr für beeinflussende Bias bewertet und dargestellt.

\section{Ergebnisse \\ $\nabla$}

Die größte Veränderung der Schmerzintensität konnte durch die Therapie mit hochzellulärem LR-PRP (LR: LeukozytenRich) erzielt werden. Ein deutlicher Vorteil eines bestimmten Produktes aus der LR-PRP Gruppe wurde nicht nachgewiesen. Die Injektion mit ACP zeigte ebenfalls einen positiven Effekt auf die Schmerzintensität. LP-PRP zeigte eine relativ geringe

\begin{tabular}{|c|c|}
\hline Therapie mit autologen Blutprodukten & Kontrolltherapien \\
\hline Autologe Vollblutinjektion: 7 Studien & Injektionen \\
\hline LR-PRP & Kortison: 6 Studien \\
\hline GPS (Biomet Biologistics): 6 Studien & $\mathrm{NaCl}: 4$ Studien \\
\hline MyCells (Kaylight Ltd): 1 Studie & Lokalanästhetikum: 2 Studien \\
\hline Prosys (Tozai Holdings Inc): 1 Studie & Punktion ohne Injektion: 4 Studien \\
\hline \multicolumn{2}{|l|}{ Unspecified Kit: 2 Studien } \\
\hline $\begin{array}{l}\text { LR-PRP mit zusätzlicher Injektion von } 10-15 \mathrm{ml} \\
\text { Lokalanästhetikum: } 1 \text { Studie }\end{array}$ & Konservatives Vorgehen \\
\hline Leukocyte-Poor PRP (LP-PRP): 1 Studie & Exzentrisches Training: 1 Studie \\
\hline Activated LP-PRP (ACP): 1 Studie & Stoßwellentherapie: 2 Studien \\
\hline
\end{tabular}

Effektivität, die etwas besser war als die Punktion ohne Injektion (Dry needling). Die Studie mit LR-PRP additiv zu 10-15 ml Lokalanästhetikum zeigte keinen positiven Effekt auf die Schmerzintensität im 3-Monats-Follow-Up. Die Punktion ohne Injektion (Dry needling) ergab den größten Effekt auf die Schmerzintensität aus der Kontrollgruppe. Dieser Effekt war jedoch deutlich geringer als die Injektion mit ACP. In absteigender Reihenfolge konnten Wirkungen auf die Reduktion der Schmerzintensität nachgewiesen werden: Dry needling > Kortisoninjektion > Infiltration mit Lokalanästhetikum $>\mathrm{NaCl}$ Injektion. Wenn nicht anders dargestellt, wurden alle Studien mit intratendinöser Injektion sowie vorheriger Injektion von 1-2 ml Lokalanästhetikum durchgeführt. Außer in 2 Studien wurde die korrekte Lage der Injektionen in allen Studien sonografisch kontrolliert. In 4 Studien wurden 2 Injektionen durchgeführt.

\section{Kommentar \\ $\nabla$}

Trotz vieler randomisierter und kontrollierter Studien zur PRP-Therapie bei Tendinopathien konnte ein eindeutiger Effekt nicht nachgewiesen werden. Der vorliegenden Meta-Analyse gelingt dieses auf Grund der erstmalig durchgeführten Spezifizierung der PRP-Produktion und Injektionsart. Die Autoren der Studie diskutieren die Relevanz möglicher Bias ausführlich. Ebenfalls wird der Vergleich zu Kontrolltherapien ausführlich erläutert. Die Kortisoninjektion zeigt sich im 3-MonatsFollow-Up ähnlich (in-)effektiv wie die Punktion ohne Injektion (Dry needling) und schneidet deutlich schlechter ab als die LR-PRP Injektion. Die Ko-Injektion von größeren Mengen Lokalanästhetikum wird durch die Autoren als wirkungshemmend diskutiert.

Zusammenfassend gibt es starke Evidenz für die Therapie mit LR-PRP bei Tendinopathie. Dabei ist die Technik der Wahl eine fächerförmige Injektion des LR-PRP unter sonografischer Kontrolle. Die vorherige oberflächliche Injektion von 1-2 ml Lokalanästhetikum wird empfohlen.

Dr. med. Hauke Horstmann Institut für Sportmedizin Medizinische Hochschule Hannover E-Mail: horstmann.hauke@mh-hannover. de 